\title{
Evaluation of Doppler Study of Umbilical Artery in Prolonged Pregnancy- A Study from Andhra Pradesh, India
}

\author{
Vanaja Gunduํㅜㄴ Ganga Devi Chikile², Geetha Kumari Ponnada ${ }^{3}$ \\ 1Department of OBG, ACSR Government Medical College, Nellore, Andhra Pradesh, India. \\ ${ }^{2}$ Department of OBG, District Hospital, Tenali, Andhra Pradesh, India. ${ }^{3}$ Department of \\ OBG, Andhra Medical College, Visakhapatnam, Andhra Pradesh, India.
}

\section{ABSTRACT}

\section{BACKGROUND}

The American College of Obstetricians and Gynecologists (ACOG) and WHO (World Health Organization) define a pregnancy continuing two weeks beyond expected date of delivery as post term pregnancy. Any pregnancy which has passed beyond the expected delivery date is defined as prolonged or post-dated pregnancy. Application of Doppler ultrasound allows for examination of blood flow direction, velocity and volume of various vessels. Doppler velocimetry of umbilical artery in post-dated pregnancy has been suggested as a means of assessing fetal wellbeing. In prolonged pregnancy, the first step of management is an accurate diagnosis and antenatal care includes accurate dating of pregnancy, fetal surveillance and the option of induction of labour or expectant management or Caesarean section. We wanted to analyse the blood flow in umbilical artery using Doppler ultrasound in post-dated pregnancy and analyse the perinatal outcome in post-dated pregnancies with respect to normal and abnormal doppler wave forms.

\section{METHODS}

This is a prospective study conducted at Department of Obstetrics and Gynaecology, Government Victoria Hospital (GVH), Visakhapatnam, from April 2016 to April 2017. 110 pregnant women who were beyond the expected date of delivery (EDD) according to menstrual history and early weeks scan were selected from the antenatal ward and labour room.

\section{RESULTS}

In the present study, $52.73 \%$ women with prolonged pregnancy were primi gravida and $67.27 \%$ women were between the gestational ages of 40 - 41 weeks. Doppler studies were abnormal in $13.64 \%$ (15 women). $78.1 \%$ women with prolonged pregnancy had normal vaginal delivery, Caesarean section was done in $17.3 \%$ and $4.6 \%$ had instrumental delivery. $99.09 \%$ of babies were live born and one was stillborn. Two babies died in early neonatal period due to meconium aspiration.

\section{CONCLUSIONS}

Doppler study of umbilical arteries is a useful noninvasive procedure to reduce the perinatal morbidity and mortality in prolonged pregnancy.

\section{KEY WORDS}

Antepartum Fetal Surveillance, Birth Asphyxia, Doppler Study, Intrauterine Death, Perinatal Outcome, Prolonged Pregnancy, Pulsatility Index, Resistance Index
Corresponding Author:

Dr. Vanaja Gundu,

Door No. 9-7-289, Sairam Nagar, Lane Opposite Vaibhav Jewelleries, Gajuwaka, Visakhapatnam - 530026, Andhra Pradesh, India.

E-mail:vanajasekhar@yahoo.com

DOI: $10.14260 /$ jemds/2021/361

How to Cite This Article:

Gundu V, Chikile GD, Ponnada GK. Evaluation of Doppler study of umbilical artery in prolonged pregnancy-A Study from Andhra Pradesh, India. J Evolution Med Dent Sci 2021;10(23):1746-1750, DOI: $10.14260 /$ jemds $/ 2021 / 361$

Submission 28-01-2021,

Peer Review 14-04-2021,

Acceptance 20-04-2021,

Published 07-06-2021.

Copyright (C) 2021 Vanaja Gundu et al. This is an open access article distributed under Creative Commons Attribution License [Attribution 4.0 International (CC BY 4.0)] 


\section{BACKGROUND}

The incidence of prolonged pregnancy is $1.1 \%$. There are many methods for antepartum fetal surveillance to evaluate the fetal well-being in prolonged pregnancy. They are daily fetal movement count by mother, ultrasound for growth, amniotic fluid index (AFI), non-stress test, Vibroacoustic stimulation test, biophysical profile (BPP), colour Doppler studies and ST segment analysis in fetal ECG (STAN). Among these evaluations of pulsatility index, resistance index and S / $D$ ratio of umbilical artery is found to be reliable.

Umbilical artery Doppler studies are often used to identify fetal compromise due to altered fetal circulation. The use of Doppler for detecting blood flow velocities was described by Johnson and Associates in 1972. Fritzgerald and Drumm described the first application of Doppler ultrasound in obstetrics in 1977. They used a $5 \mathrm{MHz}$ continuous wave Doppler device guided by a pulsed echo-B-mode ultrasonic imaging machine to assess the fetal umbilical circulation. This provided a unique non-invasive and safe method of studying blood flow characteristics in both the fetal, placental circulation and maternal arterial supply to the intervillous space.

The ratios (indices) of the resistance to blood flow i.e. impedance are calculated using the following formulae.

- S / D ratio

- Resistance index (RI) = Systolic velocity - diastolic velocity / systolic velocity

- $\quad$ Pulsatility index (PI) = Systolic velocity - diastolic velocity / mean velocity

The S / D ratio in the umbilical artery normally decreases from approximately 4.0 at 20 weeks to 2.0 at term, generally less than 3.0 after 30 weeks. Because of the downstream impedance to flow, more end-diastolic flow is observed at the placental cord insertion than at the fetal ventral wall. Thus, the abnormalities such as absent or reversed diastolic flow appear first at the fetal cord insertion.

Studies in prolonged pregnancy show the use of Doppler to predict adverse fetal outcomes like abnormal fetal heart rate, meconium aspiration, need for operative delivery have had contrasting outcomes.1,2,3 When there is absent or reversal of diastolic flow in umbilical artery, it indicates impending intra uterine fetal death. The ultimate goal of antepartum fetal surveillance (AFS) is to identify the foetuses at risk of intrauterine death (IUD) or other complications of intrauterine asphyxia and intervene to prevent these adverse outcomes if possible. ${ }^{4}$

\section{Objectives}

1. To estimate the blood flow in umbilical artery using Doppler ultrasound in post-dated pregnancy with reference to flow velocity wave form indices that is resistance index (RI), pulsatility index (PI) and systolic / diastolic (S / D) ratio.

2. To determine the efficacy of umbilical artery Doppler is an effective tool for antepartum fetal surveillance.

3. To analyse the perinatal outcome in post-dated pregnancies with respect to normal and abnormal Doppler wave forms.

\section{METHODS}

This is a prospective study conducted at Department of Obstetrics and Gynaecology, Government Victoria Hospital (GVH), Visakhapatnam from April 2016 to April 2017. 110 pregnant women who were beyond the expected date of delivery (EDD) according to menstrual history and early weeks scan were selected from the antenatal ward and labour room.

Inclusion and exclusion criteria were followed. After routine investigations, Doppler studies of umbilical artery (resistance index, pulsatility index and S / D ratio) along with Biophysical profile (BPP) was performed and the results were analysed with $P$ values and descriptive statistics and those results were compared with various studies. (110 antenatal women with prolonged pregnancies were admitted in Government Victoria hospital from April 2016 to April 2017. Hence, we took 110 women as sample size. We performed Doppler studies of umbilical artery and the variables like neonatal outcome were analysed in antenatal women with normal doppler studies and those with abnormal doppler studies with P-values and descriptive statistics)

Institutional ethical committee was obtained. Informed consent from all the women who were included the study was taken.

\section{Inclusion Criteria}

1. Women with last menstrual period (LMP) definitely known with previous regular menstrual cycles and with dates beyond EDD were included.

2. In women with definite LMP not known or previous irregular cycles, confirmation of gestational age by ultrasound examination in the early weeks has been considered.

3. They should have singleton and uncomplicated pregnancy.

4. Women who were in latent phase of labour were also included.

\section{Exclusion Criteria}

1. Women with any antenatal complications were not included in the study.

2. Women with oral contraceptive pill usage prior to this conception were excluded from the study.

3. Women in active phase of labour were excluded.

\section{Statistical Analysis}

Data entry was done using the Microsoft Excel 2010 sheet and the statistical analysis was performed with chi-square test, Fisher's exact test and Student T-test. Statistical significance was considered at $\mathrm{P}=0.05$.

\section{RESULTS}

A group of 110 women with prolonged pregnancy beyond 40 weeks of gestation were studied, ultrasound assessment of gestational age was performed, umbilical artery Doppler wave 
forms were recorded, and biophysical profile was done, and these results were correlated with the perinatal outcome.

In the present study, the booked and UN booked cases were $41.82 \%(\mathrm{~N}=46)$ and $58.18 \%(\mathrm{~N}=64)$ respectively. Most of the women were in the age group of $21-25$ years accounting for $79.09 \%(\mathrm{~N}=87) .52 .73 \%(\mathrm{~N}=58)$ of the women were primigravida, $37.27 \%(\mathrm{~N}=41)$ were second gravida and $10 \%(\mathrm{~N}=11)$ were third gravida. $67.27 \%(\mathrm{~N}=$ 74) women were in between 40 - 41 weeks of gestation, 27.27 $\%(\mathrm{~N}=30)$ of patients were between $41-42$ weeks and 5.45 $\%(\mathrm{~N}=6)$ were beyond 42 weeks of gestation. Doppler study was normal in $86.36 \%$ (95 women). Abnormal Doppler study was observed in 4 women, 8 women and 3 women with gestational ages of $40-41$ weeks, $41-42$ weeks and beyond 42 weeks respectively.

\begin{tabular}{|cc|c|}
\hline $\begin{array}{c}\text { Gestational Age } \\
\text { (Weeks) }\end{array}$ & $\begin{array}{c}\text { Normal Doppler } \\
\text { (95) }\end{array}$ & $\begin{array}{c}\text { Abnormal } \\
\text { Doppler (15) }\end{array}$ \\
$40-41(74)$ & 70 & 4 \\
$41-41(30)$ & 22 & 8 \\
$>42(6)$ & 3 & 3 \\
\hline $\begin{array}{c}\text { Percentage of normal and abnormal } \\
\text { Doppler }\end{array}$ & $86.36 \%$ & $13.64 \%$ \\
\hline Table 1. Doppler Indices in Relation to Gestational Age \\
\hline
\end{tabular}

Normal BPP was seen in $79.09 \%(\mathrm{~N}=87)$ of women and abnormal BPP was seen in $20.91 \%(\mathrm{~N}=23)$ of women in the present study. Mild oligohydramnios with Amniotic fluid Index (AFI) 5 - $8 \mathrm{~cm}$ was seen in $11.8 \%(\mathrm{~N}=13)$ of women and severe oligohydramnios of $<5 \mathrm{~cm}$ was seen in $8.18 \%(\mathrm{~N}$ = 9) women. $69.09 \%(\mathrm{~N}=76)$ of women were induced with misoprostol, $30 \%(\mathrm{~N}=33)$ attended labour room with spontaneous onset of labour pains and one woman underwent elective LSCS with CPD. $78.1 \%(\mathrm{~N}=86)$ of women had normal vaginal delivery, Caesarean section was done in $17.3 \%(\mathrm{~N}=19)$ of women and $4.6 \%(\mathrm{~N}=5)$ had instrumental delivery. Lower segment Caesarean section (LSCS) was done for failed induction in $26.3 \%(\mathrm{~N}=5)$ of women, for both abnormal Doppler and BPP in $31.5 \%(\mathrm{~N}=6)$ women, for fetal distress in $21.05 \%$ of women ( $=4)$, for big baby with CPD in $5.26 \%$ women $(\mathrm{N}=1)$.

In the present study, liquor was clear in $71.8 \%$ women $(\mathrm{N}$ $=79), 18.1 \%$ women $(\mathrm{N}=20)$ had thin meconium stained liquor and thick meconium stained liquor was seen in $10 \%$ of women $(\mathrm{N}=11) .91 \%$ of babies $(\mathrm{N}=100)$ had APGAR score $>7$ and $9 \%$ of babies $(\mathrm{N}=10)$ had APGAR score $<7.99 .09 \%$ of the babies $(\mathrm{N}=109)$ were live born and one was still born. Out of the live babies two died in early neonatal period due to meconium aspiration. 16 babies had asphyxia, 6 babies had meconium aspiration syndrome, post maturity syndrome was seen in two babies, IUGR was noticed in two babies and one baby was macrocosmic. Out of 15 women with abnormal Doppler, 7 babies (46.7\%) were born with poor APGAR score and 8 babies (53.3\%) were born with normal APGAR score. Out of 23 women with abnormal BPP, 6 babies (26.1\%) were born with poor APGAR score and 17 babies (73.9\%) had normal APGAR score. Two babies died in the early neonatal period because of meconium aspiration after admission in Neonatal Intensive care unit (NICU) on second and third postnatal day. One baby was stillborn. Two perinatal deaths occurred in women with both abnormal Doppler and abnormal BPP. There was one perinatal death with only abnormal Doppler. There were no deaths in women with normal Doppler, normal BPP and with abnormal BPP.

\begin{tabular}{|c|c|c|c|c|c|c|c|c|}
\hline \multirow{2}{*}{$\begin{array}{c}\text { Gestational Age in } \\
\text { Weeks }\end{array}$} & \multirow{2}{*}{ Perinatal Outcome } & \multicolumn{3}{|c|}{ Doppler Indices } & \multirow{2}{*}{ BPP } & \multirow{2}{*}{ AFI (cm) } & \multirow{2}{*}{ APGAR } & \multirow{2}{*}{ Remarks } \\
\hline & & S / D & RI & PI & & & & \\
\hline 41 weeks 3 days & Early neonatal death & 3.1 & 0.8 & 1.1 & $6 / 8$ & 4.9 & $4-6$ & $\begin{array}{l}\text { Delivered by forceps in } \mathrm{v} \text { / o fetal distress, baby died on } \\
\text { third postnatal day }\end{array}$ \\
\hline 42 weeks 3 days & Early neonatal death & 3.2 & 0.9 & 1.3 & $8 / 8$ & 9 & $4-6$ & $\begin{array}{l}\text { Delivered by emergency LSCS in v / o fetal distress and the } \\
\text { baby expired on second postnatal day }\end{array}$ \\
\hline 44 weeks & Still birth & 3.6 & 1.1 & 1.4 & $4 / 8$ & 2 & 0 & $\begin{array}{c}\text { Delivered by emergency LSCS in v / o severe oligoamnios } \\
\text { and non-reactive NST }\end{array}$ \\
\hline \multicolumn{9}{|c|}{ Table 2. Clinical Data of Poor Perinatal Outcome } \\
\hline
\end{tabular}

\begin{tabular}{|ccc|c|}
\hline Parameters & $\begin{array}{c}\text { No. of } \\
\text { Patients }\end{array}$ & Percentage & $\begin{array}{c}\text { Perinatal } \\
\text { Mortality }\end{array}$ \\
\hline $\begin{array}{c}\text { Normal Doppler and BPP } \\
\text { Both BPP and Doppler } \\
\text { abnormal }\end{array}$ & 79 & $71.8 \%$ & Nil \\
$\begin{array}{c}\text { Abnormal Doppler only } \\
\text { Abnormal BPP only }\end{array}$ & 8 & $6.36 \%$ & 2 \\
\hline \multicolumn{3}{|c|}{$\begin{array}{c}\text { Table 3. Perinatal Mortality Depending on } \\
\text { Both Abnormal Doppler and BPP }\end{array}$} \\
\hline
\end{tabular}

\section{DISCUSSION}

A prospective study was conducted on 110 pregnant women with prolonged pregnancy who attended the Department of Obstetrics and Gynaecology, Government Victoria Hospital (GVH) from April 2016 to April 2017 for evaluation of Doppler. One of the major goals of ante partum fetal surveillance is early detection and timely intervention. There were various methods of antepartum fetal surveillance. The best method is the one which aims at identifying the fetus at risk and requiring immediate intervention. In this study the umbilical artery doppler and biophysical profile were used for fetal surveillance in prolonged pregnancy.

The women who were admitted in antenatal ward and labour room with prolonged pregnancy were included in the study. The results were analysed with $P$ values and descriptive statistics and results were compared with various studies.

74 women (67.2\%) were in between the gestational age of $40-41$ weeks and $30(27.3 \%)$ were in between 41 to 42 weeks. 6 (5.4\%) women had gestational age of more than 42 weeks.

\begin{tabular}{|c|c|c|c|c|}
\hline $\begin{array}{c}\text { Gestational } \\
\text { Age (Weeks) }\end{array}$ & $40-41(N=74)$ & $\begin{array}{l}41-42 \\
(N=30)\end{array}$ & $\begin{array}{c}>42 \\
(N=6)\end{array}$ & Percentage \% \\
\hline $\begin{array}{c}\text { Normal Doppler } \\
(\mathrm{N}=95)\end{array}$ & $70(94.6 \%)$ & $22(73.34 \%)$ & $3(50 \%)$ & 86.36 \\
\hline \begin{tabular}{|c} 
Abnormal \\
Doppler $(\mathrm{N}=15)$ \\
\end{tabular} & $4(5.4 \%)$ & $8(26.66 \%)$ & $3(50 \%)$ & 13.64 \\
\hline \multicolumn{5}{|c|}{ Table 4. Doppler Values in Prolonged Pregnancy } \\
\hline \multicolumn{5}{|c|}{ Chi-square $=15.32, \mathrm{df}=2, \mathrm{P}$-value $=0.001 \mathrm{HS}$} \\
\hline
\end{tabular}

In the present study, 15 women (13.6 \%) have shown abnormal Doppler waveforms in umbilical artery. Out of 15 
patients, 8 cases have shown borderline abnormal Doppler Wave forms with RI value in between 0.58 and 0.62 and S / D ratio in between 2.4 and 2.8. 7 patients have shown increased abnormal waveforms with RI value greater than 0.62 and S / D ratio greater than 2.8 and out of them 5 cases have shown signs of fetal distress at birth. Out of 5 cases, one was still born and one early neonatal death due to meconium aspiration, both of them had S / D ratio > 3

Out of 6 cases, 3 patients beyond 42 weeks of gestation have shown abnormal Doppler values indicating that impedance of flow increases with gestational age.

Chi-square test of 15.32 , df of 2 , P-value $=0.001$ are highly significant suggesting strong association of abnormal Doppler with increasing gestational age. This depicts abnormal Doppler is a good predictor in anticipating poor perinatal outcome with a sensitivity of $70 \%$ and specificity of $90.5 \%$ which is consistent with the study of Zimmerman et al. ${ }^{1}$ where the sensitivity of the test is $37 \%$ and specificity is $73 \%$ and sensitivity of the test increases to $50 \%$ after taking cut off value of RI as 0.58 .

\section{Absent or Reversal of End Diastolic Flow Velocity}

Most extreme form of waveform abnormality and it represents a unique and severe fetal compromise which was not met in this study. In the present study, among 110 patients, 23 women (20.9\%) showed abnormal biophysical profile. Out of 23 patients, 22 cases had oligohydramnios of AFI below 8, 9 patients had severe oligohydramnios of less than 5 and 1 patient had non-reactive NST with late and variable decelerations and with S / D ratio of 3.3 who underwent emergency Caesarean section but had stillborn baby. 6 patients beyond 42 weeks of gestation had abnormal BPP and chi square test of 18.98 , df value 2 and with $P$ value of 0.000016 suggesting strong association of abnormal BPP with increasing gestational age.

In the present study, 34 women (30\%) had spontaneous onset of labour pains and 75 patients $(69.1 \%)$ were induced with misoprostol, one patient underwent elective LSCS with an indication of CPD. The incidence of induction of labour is comparable with the study conducted by Zimmerman et al. in which labour was induced in $76 \%$ of patients. In the present study, rate of Caesarean section was $53.3 \%$ patients with increased impedance and $7 \%$ in patients with normal impedance. The results are comparable with the study done by Hitschold et al. ${ }^{5}$ where the rate of Caesarean section was $53 \%$ in patients with increased impedance and $3 \%$ in patients with normal impedance.

\section{Colour of the Liquor}

Thick meconium stained liquor was observed in 4 out of 6 patients in gestational age group of more than 42 weeks. Chisquare test $=36.43$, df- 4 , P-value is $<0.001$ which is highly significant. This depicts that incidence of thick meconium increases with increasing gestational age. In the present study, thick meconium stained liquor was seen in $10 \%$ of the patients and the result was consistent with the study done by S.K. Patil et al. ${ }^{2}$ in that thick meconium stained liquor was $11.5 \%$. In a study by Sowmya et al. ${ }^{3}$ it is $27.14 \% 109$ (99.1\%) were live births and one baby (0.9\%) was stillborn. Out of 109 live births, 2 were early neonatal deaths due to meconium aspiration. The perinatal mortality is $2.7 \%$. In the study conducted by Eden et al. in high-risk pregnancy, the 5-minute APGAR $<7$ score observed was $1.5 \%$, in the study done by Sowmya et al. ${ }^{3}$ it was $18.5 \%$ and, in the study, done by Nageotte et al. ${ }^{6}$ it was $0.8 \%$. In the present study 5-minute APGAR score less than 7 was seen in 10 (9\%) cases.

\begin{tabular}{|ccc|}
\hline Value & Present Study & Zimmerman et al. Study \\
Sensitivity & $70 \%$ & $50 \%$ \\
Specificity & $91 \%$ & $75 \%$ \\
Positive predictive value & $46.6 \%$ & $40 \%$ \\
Negative predictive value & $96.4 \%$ & $73 \%$ \\
Validity of Doppler for Perinatal Outcome \\
Value & Present Study & Nadeemullah et al. \\
Sensitivity & $60 \%$ & $79.1 \%$ \\
Specificity & $83 \%$ & $92.9 \%$ \\
Positive predictive value & $26 \%$ & $98.5 \%$ \\
Negative predictive value & $95.4 \%$ & $41.9 \%$ \\
\hline \multicolumn{2}{c}{ Table 5. Validity of BPP for Perinatal Outcome } \\
\hline \multicolumn{2}{|c}{} \\
\hline
\end{tabular}

Out of 15 patients with abnormal Doppler, 8 patients had normal outcome and 7 patients had abnormal outcome with the sensitivity and specificity of $70 \%$ and $90 \%$ respectively indicating that Doppler is highly accurate and reliable of diagnosing fetal distress, whereas in Zimmerman et al. study the sensitivity and specificity was $50 \%$ and $75 \%$ respectively.

The sensitivity and specificity of BPP for perinatal outcome was $60 \%$ and $83 \%$ respectively, which was in consistent with the study of Nadeemullah et al. where the sensitivity and specificity were $79.1 \%$ and $92.9 \%$ respectively.

\section{CONCLUSIONS}

Doppler ultrasound was found to be a useful noninvasive tool to evaluate fetus at risk in prolonged pregnancies when available and especially when correlated with other antepartum tests such as biophysical profile. Application of BPP scoring with umbilical artery Doppler, early decision for elective Caesarean section, timely induction in favourable cases with proper intrapartum vigilance and management, help in improving the outcome in cases of prolonged pregnancy. With abnormal Doppler study, poor fetal outcome is observed with increasing gestational age. Finally, the study concludes that Doppler study is a useful noninvasive procedure to reduce the perinatal morbidity and mortality in prolonged pregnancy.

Data sharing statement provided by the authors is available with the full text of this article at jemds.com.

Financial or other competing interests: None.

Disclosure forms provided by the authors are available with the full text of this article at jemds.com.

\section{REFERENCES}

[1] Zimmermann P, Alback T, Koskinen J, et al. Doppler flow velocimetry of the umbilical artery, uteroplacetal arteries and fetal middle cerebral artery in prolonged pregnancy. Ultrasound Obstet Gynecol 1995;5(3):189-97. 
[2] Patil SK, Ghregrat RH, Khadilkar SS. Correlation of NST and AFV in antenatal fetal monitoring. J Obstet Gynecol India 1998;32(106):177-81.

[3] Sowmya KP, Mudanur SR, Padmasri R, et al. Modified biophysical profile in antepartum fetal surveillance of high risk pregnancies. Int J Reprod Contracept Obstet Gynecol 2007;6(5):1854-8.

[4] Depp R. Care of the high - risk mother. The Global Library of Women Medicine 01, 2009: DOI; 10.3843 / Glowm.10
[5] Magann EF, Sandlin AT, Songthip T. Amniotic fluid and the clinical relevance of the sonographically estimated amniotic fluid volume: oligohydramnios. J Ultrasound Med 2011;30(11)1573-85.

[6] Nageotte MP, Towers CV, Asrat T, et al. Perinatal outcome with the modified biophysical profile. Am J Obstet Gynecol 1994;170(6):1672-6. 